\title{
COMPACT HANKEL OPERATORS ON WEIGHTED HARMONIC BERGMAN SPACES
}

\author{
by KAREL STROETHOFF†
}

(Received 10 August, 1995)

\begin{abstract}
We prove the compactness of certain Hankel operators on weighted Bergman spaces of harmonic functions on the unit ball in $\mathbf{R}^{n}$.
\end{abstract}

1. Introduction. We denote the unit ball in $\mathbf{R}^{n}$ by $B_{n}$. Let $w$ be a non-negative integrable function on the interval $[0,1)$, henceforth called a weight function, and consider the weighted Bergman space $b_{w}^{2}\left(B_{n}\right)$ of harmonic functions $u$ on $B_{n}$ for which

$$
\|u\|_{w}=\left(\int_{B_{n}}|u(x)|^{2} w(|x|) d V(x)\right)^{1 / 2}<\infty,
$$

where $V$ denotes the usual Lebesgue volume measure. We shall show that under mild conditions on the weight function $w$ the space $b_{w}^{2}\left(B_{n}\right)$ is a closed linear subspace of $L_{w}^{2}\left(B_{n}\right)$, the space of all square-integrable functions on $B_{n}$ with respect to the measure $w(|x|) d V(x)$, so that there exists an orthogonal projection $Q_{w}$ of $L_{w}^{2}\left(B_{n}\right)$ onto $b_{w}^{2}\left(B_{n}\right)$. For a function $f \in L^{\infty}\left(B_{n}\right)$ define the Hankel operator $H_{f}: b_{w}^{2}\left(B_{n}\right) \rightarrow L_{w}^{2}\left(B_{n}\right)$ by

$$
H_{f} u=\left(I-Q_{w}\right)(f u), \quad u \in b_{w}^{2}\left(B_{n}\right) \text {. }
$$

The operator $H_{f}$ is clearly bounded on $b_{w}^{2}\left(B_{n}\right)$ with $\left\|H_{f}\right\| \leqslant\|f\|_{\infty}$. In this paper we prove that for every $f$ continuous on the closed unit ball $\bar{B}_{n}$ the operator $H_{f}$ is compact on $b_{w}^{2}\left(B_{n}\right)$, extending a recent result of $\mathrm{M}$. Jovovic [4] to the setting of weighted harmonic Bergman spaces.

In Section 2 we give the preliminaries for the paper. In Section 3 we discuss weighted Bergman spaces and the Bergman projection. In Section 4 we discuss Hankel operators and prove the above mentioned result. We furthermore show that these Hankel operators are in general not Hilbert-Schmidt.

2. Preliminaries. We recall that a twice-continuously differentiable function $u$ on $B_{n}$ is harmonic on $B_{n}$ if $\Delta u \equiv 0$, where $\Delta=D_{1}^{2}+\ldots+D_{n}^{2}$ and $D_{j}$ denotes the partial derivative with respect to the $j$-th coordinate. A polynomial on $\mathbf{R}^{n}$ is homogeneous of degree $m$ (or $m$-homogeneous) if it is a finite linear combination of monomials $x_{1}^{\alpha_{1}} \ldots x_{n}^{\alpha_{n}}$, where $\alpha_{1}, \ldots, \alpha_{n}$ are nonnegative integers such that $\alpha_{1}+\ldots+\alpha_{n}=m$. It is easy to show that a polynomial $p$ on $\mathbf{R}^{n}$ is homogeneous of degree $m$ if and only if $x \cdot \nabla p(x)=m p(x)$ for all $x \in \mathbf{R}^{n}$, where $\nabla$ denotes the gradient. Every harmonic function $u$ on $B_{n}$ can be decomposed as $u=\sum_{k=0}^{\infty} u_{k}$, where each $u_{k}$ is a harmonic homogeneous polynomial of degree $k$, and the convergence is uniform on compact subsets of $B_{n}$. Denote the unit sphere in $\mathbf{R}^{n}$ by $S_{n}$. The space $\mathscr{H}_{k}\left(S_{n}\right)$ of restrictions to $S_{n}$ of harmonic homogeneous

† The author was partially supported by grants from the Montana University System and the University of Montana.

Glasgow Math. J. 39 (1997) 77-84. 
polynomials of degree $k$, the so-called spherical harmonics of degree $k$, is a (finitedimensional) Hilbert space with respect to the usual inner product on $L^{2}\left(S_{n}, d \sigma\right)$, where $\sigma$ denotes the normalized surface-area measure on $S_{n}$. For each $\eta \in S_{n}$ the linear functional $p \mapsto p(\eta)$ on the space $\mathscr{H}_{k}\left(S_{n}\right)$ is uniquely represented by a harmonic $k$-homogeneous polynomial $Z_{k}(\cdot, \eta)$, called the zonal harmonic of degree $k$ at $\eta$. Extending $Z_{k}$ to a function on $\mathbf{R}^{n} \times \mathbf{R}^{n}$ by setting $Z_{k}(x, y)=|y|^{k} Z_{k}(x, y /|y|)$, and using the fact that each zonal harmonic $Z_{k}(\cdot, \eta)$ is real valued (see pages $78-79$ in [1]) we have

$$
\int_{S_{n}} p(\zeta) Z_{k}(\zeta, y) d \sigma(\zeta)=p(y)
$$

for every harmonic $k$-homogeneous polynomial $p$. Denoting the dimension of $\mathscr{L}_{k}\left(S_{n}\right)$ by $h_{k}$, it is easily seen that $Z_{k}(\eta, \eta)=h_{k}$, for all $\eta \in S_{n}$, and thus $Z_{k}(y, y)=|y|^{2 k} h_{k}$, for all $y \in \mathbf{R}^{n}$.

Spherical harmonics of distinct degrees are orthogonal; that is,

$$
\int_{S_{n}} p \bar{q} d \sigma=0
$$

if $p$ and $q$ are harmonic homogeneous polynomials of distinct degree.

In the sequel the following theorem will play an important role.

THEOREM 2.2. (Spherical Decomposition Theorem.) If $p$ is a homogeneous polynomial of degree $m$, then for each $k=0,1, \ldots,[\mathrm{m} / 2]$ there exist a harmonic homogeneous polynomial $p_{m-2 k}$ of degree $m-2 k$, such that

$$
p(x)=\sum_{k=0}^{[m / 2]}|x|^{2 k} p_{m-2 k}(x) \text {. }
$$

A constructive proof of the above theorem has recently been given in [3]. We observe that another constructive proof may be given as follows. It is elementary to show that for a harmonic $j$-homogeneous polynomial $q$ we have

$$
\Delta\left[|x|^{2 i} q\right]=2 i(n+2 j+2 i-2)|x|^{2 i-2} q .
$$

Assuming that $\sum_{k=0}^{[m / 2]-1}|x|^{2 k} q_{m-2 k-2}$ is the spherical decomposition of $\Delta p$, it follows with the help of (2.3) that

$$
\begin{aligned}
\Delta\left[\sum_{k=1}^{[m / 2]}|x|^{2 k} \frac{q_{m-2 k}}{2 k(n+2 m-2 k-2)}\right] & =\sum_{k=1}^{[m / 2]}|x|^{2 k-2} q_{m-2 k} \\
& =\sum_{k=0}^{[m / 2]-1}|x|^{2 k} q_{m-2 k-2}=\Delta p,
\end{aligned}
$$

so that

$$
p_{m}=p-\sum_{k=1}^{\mid m / 2]}|x|^{2 k} \frac{q_{m-2 k}}{2 k(n+2 m-2 k-2)}
$$

is a harmonic $m$-homogeneous polynomial, and thus $p=\sum_{k=0}^{[m / 2]}|x|^{2 k} p_{m-2 k}$ is the spherical 
decomposition of $p$, where $p_{m-2 k}=q_{m-2 k} /(2 k(n+2 m-2 k-2))$ for $k \geq 1$. We shall use this idea in Section 4 to find explicit formulae for the norms of the Hankel operators associated with the coordinate functions.

3. Weighted harmonic Bergman spaces. For a weight function $w$ we introduce the moments

$$
\hat{w}(k)=\int_{B_{n}}|x|^{k} w(|x|) d V(x), \quad(k=0,1, \ldots) .
$$

We shall assume that $\hat{w}(k)>0$, for all $k=0,1, \ldots$ If $p$ and $q$ are homogeneous harmonic polynomials of degrees $k$ and $l$ respectively then, integrating in polar coordinates, it is easily seen that

$$
\langle p, q\rangle_{w}= \begin{cases}\hat{w}(2 k) \int_{S_{n}} p \bar{q} d \sigma, & \text { if } k=l \\ 0 & \text { otherwise. }\end{cases}
$$

If $u \in b_{w}^{2}\left(B_{n}\right)$ has decomposition $u=\sum_{k=0}^{\infty} u_{k}$, where each $u_{k}$ is an harmonic $k$ homogeneous polynomial, then it follows from (2.1) and (3.1) that

$$
u_{k}(y)=\frac{1}{\hat{w}(2 k)}\left\langle u_{k}, Z_{k}(\cdot, y)\right\rangle_{w} .
$$

In particular,

$$
\left\|Z_{k}(\cdot, y)\right\|_{w}^{2}=\left\langle Z_{k}(\cdot, y), Z_{k}(\cdot, y)\right\rangle_{w}=\hat{w}(2 k) Z_{k}(y, y)=\hat{w}(2 k) h_{k}|y|^{2 k} .
$$

Applying the Cauchy-Schwarz inequality we obtain

and it follows that

$$
\left|u_{k}(y)\right| \leq(1 / \hat{w}(2 k))\left\|u_{k}\right\|_{w}\left\|Z_{k}(\cdot, y)\right\|_{w},
$$

$$
\begin{aligned}
|u(y)| & \leq \sum_{k=0}^{\infty} \frac{1}{\hat{w}(2 k)}\left\|u_{k}\right\|_{w}\left\|Z_{k}(\cdot, y)\right\|_{w} \\
& \leq\left(\sum_{k=0}^{\infty}\left\|u_{k}\right\|_{w}^{2}\right)^{1 / 2}\left(\sum_{k=0}^{\infty} \frac{h_{k}}{\hat{w}(2 k)}|y|^{2 k}\right)^{1 / 2} .
\end{aligned}
$$

We conclude that

$$
|u(y)| \leq\|u\|_{w}\left(\sum_{k=0}^{\infty} \frac{h_{k}}{\hat{w}(2 k)}|y|^{2 k}\right)^{1 / 2} .
$$

The numbers $h_{k}$ can be expressed in terms of binomial coefficients (see page 82 or 92 in [1]), and it is easily shown that $h_{k} \approx k^{n-2}$ as $k \rightarrow \infty$. The series $\sum_{k=0}^{\infty}\left(h_{k} / \hat{w}(2 k)\right)|y|^{2 k}$ has radius of convergence equal to 1 , and thus converges uniformly for $|y| \leq r<1$, for each $0<r<1$, if

$$
\limsup _{k \rightarrow \infty} 1 / \sqrt[2 k]{\hat{w}(2 k)}=1
$$

It follows from (3.2) that $b_{w}^{2}\left(B_{n}\right)$ is a closed subspace of $L_{w}^{2}\left(B_{n}\right)$ if the weight function 
satisfies (3.3). Using Exercise 3.4 of [5] it is easily shown that condition (3.3) is equivalent to the requirement that, for all $0<\delta<1$, the set $\{r \in(\delta, 1): w(r)>0\}$ has positive measure. In the sequel we assume that this condition is satisfied, so that $b_{w}^{2}\left(B_{n}\right)$ is a closed linear subspace of $L_{w}^{2}\left(B_{n}\right)$.

Furthermore, by uniform convergence and orthogonality of homogeneous harmonic polynomials of distinct degree, for each $0<r<1$ we have

$$
\int_{S_{n}}|u(r \zeta)|^{2} d \sigma(\zeta)=\sum_{k=0}^{\infty} \int_{S_{n}}\left|u_{k}(r \zeta)\right|^{2} d \sigma(\zeta),
$$

and integrating in polar coordinates we obtain

$$
\|u\|_{w}^{2}=\sum_{k=0}^{\infty}\left\|u_{k}\right\|_{w}^{2}
$$

Applying formula (3.4) to the function $u-\sum_{k=0}^{m} u_{k}=\sum_{k=m+1}^{\infty} u_{k}$ we obtain

$$
\left\|u-\sum_{k=0}^{m} u_{k}\right\|_{w}^{2}=\sum_{k=m+1}^{\infty}\left\|u_{k}\right\|_{w}^{2}
$$

Thus $\sum_{k=0}^{m} u_{k} \rightarrow u$ in $b_{w}^{2}\left(B_{n}\right)$ as $m \rightarrow \infty$. Hence the harmonic polynomials are dense in $b_{n}^{2}\left(B_{n}\right)$.

Also, if $p$ and $q$ are harmonic homogeneous polynomials of degrees $k$ and $l$, respectively, then

and thus

$$
\begin{aligned}
\left\langle|x|^{2 j} p, q\right\rangle_{w} & =n V(B) \int_{0}^{1} r^{n+2 k+2 j-1} w(r) d r \int_{S_{n}} p \bar{q} d \sigma \\
& =\hat{w}(2 k+2 j) \int_{S_{n}} p \bar{q} d \sigma
\end{aligned}
$$

$$
\left\langle|x|^{2 j} p, q\right\rangle_{w}=\frac{\hat{w}(2 k+2 j)}{\hat{w}(2 k)}\langle p, q\rangle_{w} .
$$

It follows from (3.5) and the fact that the harmonic polynomials are dense in $b_{w}^{2}\left(B_{n}\right)$ that

$$
Q_{w}\left[|x|^{2 j} p\right]=\frac{\hat{w}(2 k+2 j)}{\hat{w}(2 k)} p,
$$

for every harmonic homogeneous polynomial $p$ of degree $k$.

The following result shows that the Bergman projection of a polynomial is a harmonic polynomial of degree less than or equal to that of the original polynomial.

THEOREM 3.7. If an $m$-homogeneous polynomial $p$ has spherical decomposition given by $p(x)=\sum_{k=0}^{[m / 2]}|x|^{2 k} p_{m-2 k}(x)$, then

$$
Q_{w}[p]=\sum_{k=0}^{[m / 2]} \frac{\hat{w}(2 m-2 k)}{\hat{w}(2 m-4 k)} p_{m-2 k}
$$


Proof. If $p=\sum_{k=0}^{[m / 2]}|x|^{2 k} p_{m-2 k}$ is the spherical decomposition of $p$, then by linearity and (3.6)

$$
Q_{w}[p]=\sum_{k=0}^{[m / 2]} Q_{w}\left[|x|^{2 k} p_{m-2 k}\right]=\sum_{k=0}^{[m / 2]} \frac{\hat{w}(2 m-2 k)}{\hat{w}(2 m-4 k)} p_{m-2 k}
$$

proving the result.

Corollary 3.8. Let $w(r)=\left(1-r^{2}\right)^{\lambda}$, where $-1<\lambda<\infty$. If an $m$-homogeneous polynomial $p$ has spherical decomposition given by $p(x)=\sum_{k=0}^{[m / 2]}|x|^{2 k} p_{m-2 k}(x)$, then the projection $Q_{\lambda}[p]$ of $p$ onto $b_{w}^{2}\left(B_{n}\right)$ is given by

$$
Q_{\lambda}[p]=\sum_{k=0}^{[m / 2]} \prod_{j=1}^{k} \frac{n+2(m-2 k)+2 j-2}{n+2(m-2 k)+2 j+2 \lambda} p_{m-2 k} .
$$

Proof. An elementary calculation shows that

$$
\hat{w}(2 j)=\frac{n}{2} V\left(B_{n}\right) \frac{\Gamma\left(\frac{n}{2}+j\right) \Gamma(\lambda+1)}{\Gamma\left(j+\frac{n}{2}+\lambda+1\right)},
$$

and thus

$$
\hat{w}(2 j)=\frac{n+2 j-2}{n+2 j+2 \lambda} \hat{w}(2 j-2)
$$

for $j \geq 1$. This implies that

$$
\frac{\hat{w}(2 m-2 k)}{\hat{w}(2 m-4 k)}=\prod_{j=1}^{k} \frac{\hat{w}(2 m-4 k+2 j)}{\hat{w}(2 m-4 k+2 j-2)}=\prod_{j=1}^{k} \frac{n+2(m-2 k)+2 j-2}{n+2(m-2 k)+2 j+2 \lambda},
$$

and the stated result follows from the above theorem.

Remarks. 1. Note that as $\lambda \rightarrow-1^{+}, Q_{\lambda}[p]$ converges to the Poisson integral of $p: \sum_{k=0}^{[m / 2]} p_{m-2 k}$.

2. If $\lambda=0$, then

$$
Q_{0}[p]=\sum_{k=0}^{[m / 2]} \frac{n+2 m-4 k}{n+2 m-2 k} p_{m-2 k}
$$

as in [3].

4. Hankel operators. Let $w$ be a weight function satisfying condition (3.3). We shall consider the Hankel operator $H_{x_{1}}$ on $b_{w}^{2}\left(B_{n}\right)$. Let $p$ be a harmonic $m$-homogeneous polynomial on $\mathbf{R}^{n}$, where $m \geq 1$. Then $\Delta\left(x_{1} p\right)=2 D_{1} p(x)$. Since $x_{1} p$ is homogeneous of degree $m+1$, it follows that $x_{1} p$ has spherical decomposition given by

$$
x_{1} p=p_{m+1}+|x|^{2} p_{m-1},
$$


with

$$
p_{m-1}(x)=\frac{1}{n+2 m-2} D_{1} p(x), \quad \text { and } \quad p_{m+1}(x)=x_{1} p(x)-|x|^{2} p_{m-1}(x)
$$

Consequently

$$
\begin{aligned}
Q_{w}\left[x_{1} p\right] & =p_{m+1}+\frac{\hat{w}(2 m)}{\hat{w}(2 m-2)} p_{m-1} \\
& =x_{1} p-|x|^{2} \frac{1}{n+2 m-2} D_{1} p+\frac{\hat{w}(2 m)}{(n+2 m-2) \hat{w}(2 m-2)} D_{1} p .
\end{aligned}
$$

Hence

$$
H_{x_{1}} p=\frac{1}{n+2 m-2}\left\{|x|^{2} D_{1} p-\frac{\hat{w}(2 m)}{\hat{w}(2 m-2)} D_{1} p\right\} .
$$

If $q$ is a harmonic homogeneous polynomial of degree $k$, then

$$
\begin{aligned}
\left\langle H_{x_{1}} p, H_{x_{1}} q\right\rangle_{w} & =\left\langle H_{x_{1}} p, x_{1} q\right\rangle_{w} \\
& =\frac{1}{n+2 m-2}\left\{\left\langle|x|^{2} D_{1} p, x_{1} q\right\rangle_{w^{\prime}}-\frac{\hat{w}(2 m)}{\hat{w}(2 m-2)}\left\langle D_{1} p, x_{1} q\right\rangle_{w^{\prime}}\right\} \\
& =\frac{1}{n+2 m-2}\left\{\left\langle x_{1} D_{1} p,|x|^{2} q\right\rangle_{w}-\frac{\hat{w}(2 m)}{\hat{w}(2 m-2)}\left\langle x_{1} D_{1} p, q\right\rangle_{w^{\prime}}\right\} .
\end{aligned}
$$

Similar formulae hold for $\left\langle H_{x_{j}} p, H_{x, q} q\right\rangle_{w},(j=2, \ldots, n)$. Adding these formulae, and making use of $\sum_{j=1}^{n} x_{j} D_{j} p=m p$, we obtain

$$
\sum_{i=1}^{n}\left\langle H_{x} p, H_{x}, q\right\rangle_{w}=\frac{m}{n+2 m-2}\left\{\left\langle p,|x|^{2} q\right\rangle_{w}-\frac{\hat{w}(2 m)}{\hat{w}(2 m-2)}\langle p, q\rangle_{w}\right\} .
$$

It follows that

$$
\begin{aligned}
\sum_{j=1}^{n}\left\langle H_{x_{j}} p, H_{x} q\right\rangle_{w} & =\frac{m}{n+2 m-2}\left\{\left\langle|x|^{2} p, q\right\rangle_{w}-\frac{\hat{w}(2 m)}{\hat{w}(2 m-2)}\langle p, q\rangle_{w}\right\} \\
& =\frac{m}{n+2 m-2}\left\{\frac{\hat{w}(2 m+2)}{\hat{w}(2 m)}\langle p, q\rangle_{w}-\frac{\hat{w}(2 m)}{\hat{w}(2 m-2)}\langle p, q\rangle_{w}\right\} \\
& =\frac{m}{n+2 m-2}\left\{\frac{\hat{w}(2 m+2)}{\hat{w}(2 m)}-\frac{\hat{w}(2 m)}{\hat{w}(2 m-2)}\right\}\langle p, q\rangle_{w} .
\end{aligned}
$$

It is easy to prove that the operators $H_{x_{1}}, \ldots, H_{x_{n}}$ are unitarily equivalent on $b_{w}^{2}\left(B_{n}\right)$. In fact, if $1<j \leq n$ and $U_{j}$ is the mapping defined on $L_{w}^{2}\left(B_{n}\right)$ by $\left(U_{j} g\right)(x)=g(\tilde{x})$, where $\tilde{x}$ is the vector obtained from $x$ by interchanging its first and $j$ th coordinate, then $U_{j}$ is a unitary operator on $L_{w}^{2}\left(B_{n}\right)$ mapping $b_{w}^{2}\left(B_{n}\right)$ into itself, and $H_{x_{j}} U_{j} g=U_{j} H_{x_{1}} g$, for all $g \in b_{w}^{2}\left(B_{n}\right)$ (which is easily verified by using (4.1) and the analogous formula for $H_{x_{j}}$ ). In particular, we have

$$
\left\|H_{x_{1}} p\right\|_{w}^{2}=\frac{m}{n(n+2 m-2)}\left(\frac{\hat{w}(2 m+2)}{\hat{w}(2 m)}-\frac{\hat{w}(2 m)}{\hat{w}(2 m-2)}\right)\|p\|_{w}^{2},
$$


for every harmonic $m$-homogeneous polynomial $p$ with $m \geq 1$.

Note that (4.2) implies that $\hat{w}(2 m+2) / \hat{w}(2 m) \geq \hat{w}(2 m) / \hat{w}(2 m-2)$, which can also be verified directly using the Cauchy-Schwarz inequality: also $\hat{w}(2 m)^{2} \leq$ $\hat{w}(2 m-2) \hat{w}(2 m+2)$. It follows from (3.3) that $\lim _{m \rightarrow \infty} \hat{w}(2 m+2) / \hat{w}(2 m)=1$. That $H_{x_{1}}$ is compact on $b_{w}^{2}(B)$ is proved as follows. Write $\mathscr{V}_{k}$ for the space of all harmonic polynomials of degree at most $k$. Let $S_{k}$ denote the operators defined on $b_{w}^{2}\left(B_{n}\right)$ such that $S_{k} p=H_{x_{1}} p$ if $p \in \mathscr{V}_{k}$ and $S_{k} p=0$ if $p \in b_{w}^{2}(B) \ominus \mathscr{V}_{m}$. We shall estimate $\left\|H_{x_{1}}-S_{k}\right\|$. Write $u=\sum_{m=0}^{\infty} u_{m}$, where each $u_{m}$ is a harmonic $m$-homogeneous polynomial. Then, using (4.2), Cauchy-Schwarz and (3.4), we have

$$
\begin{aligned}
\left\|\left(H_{x_{1}}-S_{k}\right) u\right\|_{w} & \leq \sum_{m=k+1}^{\infty}\left\|H_{x_{1}} u_{m}\right\|_{w} \\
& \leq \sum_{m=k+1}^{\infty}\left\{\frac{m}{n(n+2 m-2)}\left(\frac{\hat{w}(2 m+2)}{\hat{w}(2 m)}-\frac{\hat{w}(2 m)}{\hat{w}(2 m-2)}\right)\right\}^{1 / 2}\left\|u_{m}\right\|_{w} \\
& \leq \frac{1}{2}\left\{\sum_{m=k+1}^{\infty}\left(\frac{\hat{w}(2 m+2)}{\hat{w}(2 m)}-\frac{\hat{w}(2 m)}{\hat{w}(2 m-2)}\right)\right\}^{1 / 2}\left\{\sum_{m=k+1}^{\infty} \mid u_{m} \|_{w}^{2}\right\}^{1 / 2} \\
& \leq \frac{1}{2}\left\{\sum_{m=k+1}^{\infty}\left(\frac{\hat{w}(2 m+2)}{\hat{w}(2 m)}-\frac{\hat{w}(2 m)}{\hat{w}(2 m-2)}\right)\right\}^{1 / 2}\|u\|_{w},
\end{aligned}
$$

Hence

$$
\begin{aligned}
\left\|H_{x_{1}}-S_{k}\right\| & \leq \frac{1}{2}\left\{\sum_{m=k+1}^{\infty}\left(\frac{\hat{w}(2 m+2)}{\hat{w}(2 m)}-\frac{\hat{w}(2 m)}{\hat{w}(2 m-2)}\right)\right\}^{1 / 2} \\
& \leq\left(1-\rho_{k}\right)^{1 / 2}
\end{aligned}
$$

where $\rho_{k}=\hat{w}(2 k+2) / \hat{w}(2 k)$, and it follows that $S_{k} \rightarrow H_{x_{1}}$ as $k \rightarrow \infty$. Since each of the $S_{k}$ is of finite rank, the operator $H_{x_{1}}$ must be compact on $b_{n}^{2}\left(B_{n}\right)$. In fact, we have the following result.

THEOREM 4.3. Let $w$ be a weight function satisfying (3.3). Then, for every $f$ in $C\left(\bar{B}_{n}\right)$, the Hankel operator $H_{f}$ is compact on $b_{w}^{2}\left(B_{n}\right)$.

Proof. That $\mathscr{A}=\left\{f \in C\left(\bar{B}_{n}\right): H_{f}\right.$ is compact on $\left.b_{w}^{2}\left(B_{n}\right)\right\}$ is a closed algebra can be proved by the same argument as given in [2]. We have just shown that $H_{x_{1}}$ is compact on $b_{u}^{2}\left(B_{n}\right)$ and, since each of the operators $H_{x_{j}}$ is unitarily equivalent to $H_{x_{1}}$, we conclude that $x_{j} \in \mathscr{A}$, for each $j$. This implies that $\mathscr{A}$ contains all polynomials and by the Stone-Weierstrass Theorem $\mathscr{A}=C\left(\bar{B}_{n}\right)$.

It is interesting to note that the Hankel operator $H_{x_{1}}$ is in general not HilbertSchmidt. In fact, we have the following result, similar to the situation on the weighted Bergman spaces of analytic functions on the unit ball in $\mathbb{C}^{n}$. (See [6].) It shows that for $n>2$ the Hankel operator $H_{x_{1}}$ is not Hilbert-Schmidt on $b_{w}^{2}\left(B_{n}\right)$ for the indicated weight functions $w$.

TheOREM 4.4. Let $w(r)=\left(1-r^{2}\right)^{\lambda}$, where $-1<\lambda<\infty$. Then $H_{x_{1}}$ does not belong to the Schatten $\gamma$-class of $b_{w}^{2}\left(B_{n}\right)$ if $\gamma \leq n-1$. 
Proof. For $2 \leq y<\propto$ we have the inequality

$$
\left\langle\left(H_{x_{1}}^{*} H_{x_{1}}\right)^{\gamma / 2} p, p\right\rangle_{w} \geq\left\langle H_{x_{1}}^{*} H_{x_{1}} p, p\right\rangle_{w}^{\gamma / 2},
$$

for every $p \in b_{w}^{2}\left(B_{n}\right)$ of unit norm (by Proposition 6.3.3 in [7]), and it follows from (4.2) that

$$
\left\langle\left(H_{x_{1}}^{*} H_{x_{1}}\right)^{\gamma / 2} p, p\right\rangle_{w} \geq\left\{\frac{m}{n(n+2 m-2)}\left(\frac{\hat{w}(2 m+2)}{\hat{w}(2 m)}-\frac{\hat{w}(2 m)}{\hat{w}(2 m-2)}\right)\right\}^{\gamma / 2},
$$

for every $p \in b_{w}^{2}\left(B_{n}\right)$ of unit norm. Summing over an orthonormal set $h_{m}$ of $m$ homogeneous harmonic polynomials, and subsequently summing over all $m \geq 1$ we obtain

$$
\begin{aligned}
\left\|H_{x_{1}}\right\|_{\gamma}^{\gamma} & =\operatorname{trace}\left(\left(H_{x_{1}}^{*} H_{x_{1}}\right)^{\gamma / 2}\right) \\
& \geq \sum_{m=1}^{\infty}\left\{\frac{m}{n(n+2 m-2)}\left(\frac{\hat{w}(2 m+2)}{\hat{w}(2 m)}-\frac{\hat{w}(2 m)}{\hat{w}(2 m-2)}\right)\right\}^{\gamma / 2} h_{m} .
\end{aligned}
$$

Using (3.9) we have

$$
\left\|H_{x_{1}}\right\|_{\gamma}^{\gamma} \geq \sum_{m=1}^{\infty}\left\{\frac{4(\lambda+1) m}{n(n+2 m-2)(n+2 m+2 \lambda+2)(n+2 m+2 \lambda)}\right\}^{\gamma / 2} h_{m} .
$$

Since $h_{m} \approx m^{n-2}$, the assumption that $H_{x_{1}}$ belongs to the Schatten $\gamma$-class, implies that $\sum_{m=1}^{\infty} m^{n-2-\gamma}<\infty$, and thus $\gamma>n-1$.

ACKNowledgements. This article was written while visiting the Free University, Amsterdam, The Netherlands, on sabbatical leave. I thank the University of Montana for awarding me a sabbatical and the Mathematics Department of the Free University for its hospitality and support.

\section{REFERENCES}

1. S. Axler, P. Bourdon, and W. Ramey, Harmonic function theory, Graduate Texts in Mathematics (Springer-Verlag, 1992).

2. S. Axler, J. B. Conway, and G. McDonald, Toeplitz operators on Bergman spaces, Canad. J. Math. 34 (1982), 466-483.

3. S. Axler and W. Ramey, Harmonic polynomials and Dirichlet-type problems, Proc. Amer. Math. Soc. 123 (1995), 3765-3773.

4. M. Jovović, Compact Hankel operators on the harmonic Bergman space Integral Equations Operator Theory 22 (1995), 295-304.

5. W. Rudin, Real and complex analysis (McGraw-Hill, 1970).

6. K. Zhu, Schatten class Hankel operators on the Bergman space of the unit ball, Amer. J. Math. 113 (1991), 147-167.

7. K. Zhu, Operator theory in function spaces (Marcel Dekker, 1990).

\section{Department of Mathematical Sciences}

UNIVERSITY OF MONTANA

Missoula

MT 59812-1032, USA

e-mail:ma_kms@selway.umt.edu 